\title{
Neutrophil-to-Lymphocyte and Platelet-to- Lymphocyte Ratios as Noninvasive Predictors of the Therapeutic Outcomes of Systemic Corticosteroid Therapy in Ulcerative Colitis
}

\author{
Katsuya Endo Tomonori Satoh Yuki Yoshino Shiho Kondo Yoko Kawakami \\ Tomofumi Katayama Yoshiteru Sasaki Atsuko Takasu Takayuki Kogure \\ Morihisa Hirota Takayoshi Meguro Kennichi Satoh \\ Division of Gastroenterology, Tohoku Medical and Pharmaceutical University, Sendai, Japan
}

\author{
Keywords \\ Ulcerative colitis · Corticosteroid · Neutrophil/lymphocyte \\ ratio $\cdot$ Platelet/lymphocyte ratio
}

\begin{abstract}
Introduction: Predictive biomarkers for the therapeutic outcome of induction therapy with systemic corticosteroid for active ulcerative colitis (UC) have not been established. This study aimed to investigate whether neutrophil-to-lymphocyte ratio (NLR) and/or platelet-to-lymphocyte ratio (PLR) can be predictive biomarkers for the therapeutic outcomes of systemic corticosteroid therapy in UC. Methods: This was a single-center retrospective cohort study. In total, 48 patients with UC who received induction therapy with systemic corticosteroid were enrolled. Based on the achievement of clinical remission after 8 weeks of treatment, the patients were divided into the remission group $(n=28)$ and the nonremission group $(n=20)$. Clinical characteristics, NLR, and PLR at baseline between the remission and nonremission groups were compared via a univariate analysis. The independent risk factors of nonremission were identified via a multivariate analysis. Results: The baseline Mayo score, platelet count, lymphocyte count, C-reactive protein (CRP)
\end{abstract}

levels, NLR, and PLR between the 2 groups significantly differed. The nonremission group had higher NLR and PLR than the remission group (4.70 [3.04-11.3] vs. 3.10 [1.36-16.42]; $p$ $<0.05$, and 353.6 [220.3-499.8] vs. 207.2 [174.4-243.6]; $p<$ 0.001 , respectively). A multivariate analysis revealed that a Mayo score of $\geq 9$, CRP level of $\geq 1.26 \mathrm{mg} / \mathrm{dL}$, and PLR of $\geq 262$ (hazard ratio: $23.1,95 \%$ confidence interval: 1.29-413.7, $p=$ 0.033 ) were considered independent risk factors for nonremission. Conclusion: This report first identified the efficacy of NLR and PLR as candidate biomarkers for predicting the therapeutic outcomes of systemic corticosteroid therapy in UC.

(c) 2021 The Author(s).

Published by S. Karger AG, Basel

\section{Introduction}

Ulcerative colitis (UC) is a chronic idiopathic inflammatory bowel disease that affects the colonic and rectal mucosa. The initial treatment for active mild or moderate UC is topical and/or systemic administration of 5-aminosalicylic acid. Systemic corticosteroids can be used in patients with moderate to severe disease activity. Prednisolone (PSL) is a common systemic corticosteroid therapy,

Correspondence to:

Katsuya Endo, kendo@med.tohoku.ac.jp

karger@karger.com www.karger.com/iid

Karger $\stackrel{\text { ' }}{5}$

BOPEN ACCESS
2021 The Author(s).

Published by S. Karger AG, Basel

This is an Open Access article licensed under the Creative Commons Attribution-NonCommercial-4.0 International License (CC BY-NC) (http://www.karger.com/Services/OpenAccessLicense), applicable to the online version of the article only. Usage and distribution for commercial purposes requires written permission. 
and its starting doses are $40 \mathrm{mg} /$ day for active moderate UC and $60 \mathrm{mg} /$ day for active severe UC [1]. The recommended tapering strategy for PSL is reducing the daily dose by $5 \mathrm{mg}$ per week, resulting in a treatment course of 8 weeks [2]. More than $80 \%$ of patients respond to systemic corticosteroid therapy, and about $55 \%$ of patients can achieve complete remission within 30 days. Meanwhile, about $45 \%$ present with partial or no response to treatment [3]. However, response to systemic corticosteroids is challenging to predict before treatment. Moreover, predictive biomarkers for the therapeutic outcome of systemic steroids have not been established. Hence, further studies should be conducted.

Previous studies have focused on the efficacy of neutrophil-to-lymphocyte ratio (NLR) and platelet-to-lymphocyte ratio (PLR) as noninvasive biomarkers for assessing the disease activity of UC [4-9]. NLR and/or PLR are significantly correlated with both clinical and endoscopic disease activity in patients with UC. Recently, several studies have shown that NLR and/or PLR can be simple biomarkers for predicting the therapeutic outcomes of UC treatment. Nishida et al. $[10,11]$ have shown that NLR can be a possible predictor of nonresponse to infliximab and for clinical relapse after tacrolimus induction in patients with UC. Bertani et al. [12] have revealed that NLR and PLR can predict mucosal healing in patients with UC treated with anti-TNF antibodies. However, only few studies have examined the relationship between pretreatment NLR and PLR and the therapeutic outcomes of induction therapy with systemic corticosteroid in UC. Therefore, the current study aimed to determine whether NLR and PLR can be predictive biomarkers for the therapeutic outcomes of systemic corticosteroid therapy in UC.

\section{Methods}

\section{Study Design and Patients}

This was a single-center retrospective cohort study, and it was approved by the ethics committee of our institution (Institutional Review Board of the Tohoku Medical and Pharmaceutical University School of Medicine). Approved IRB number is 2021-2-036. We enrolled 48 consecutive patients with active UC who received remission induction therapy via the systemic administration of corticosteroid between January 2017 and December 2020. Systemic administration of corticosteroid was defined as induction therapy with a PSL starting dose of $>30 \mathrm{mg} /$ body. The administration route and the dose of PSL were determined based on disease activity and body weight. For the outpatient with moderate disease activity, PSL was administrated orally at a dose of $30-40 \mathrm{mg} / \mathrm{body}$. For the inpatient with moderate to severe disease activity, intravenous PSL was started at a dose of $40 \mathrm{mg}-60 \mathrm{mg} /$ body. All patients were not treated with concomitant immunomodulators, calcineurin inhibitors, biologics, and tofacitinib at baseline. Data about sex, age, disease extension, disease duration, Mayo score, and laboratory findings at baseline were collected from the patients' medical records. NLR at baseline was calculated by dividing the absolute neutrophil count by the absolute lymphocyte count. PLR at baseline was calculated by dividing the absolute platelet count by the absolute lymphocyte count.

Comparisons between the Remission and Nonremission Groups after 8 Weeks of Treatment

Information about the achievement of clinical remission 8 weeks after the start of systemic steroid therapy was obtained from the patients' medical records. Clinical remission was defined as a partial Mayo score of 0 (without the endoscopic subscore). The patients were divided into the remission and nonremission groups based on the achievement of clinical remission after 8 weeks of treatment. Patients with a partial Mayo score of 0 after 8 weeks were included in the remission group. Meanwhile, patients with a partial Mayo score of $\geq 1$ after 8 weeks of treatment and those who were steroid refractory and who required additional treatment or change of drug regimen within 8 weeks were classified under the nonremission group. Clinical characteristics, NLR, and PLR at baseline between the remission and nonremission groups were compared via a univariate analysis. For continuous variables that were statistically different between the 2 groups, the optimal cutoff values for nonremission were determined via a receiver operating characteristic (ROC) analysis.

\section{Risk Factors for Predicting Nonremission after 8 Weeks of}

Treatment

To identify the independent risk factors of nonremission after 8 weeks of treatment, we performed a multivariate analysis of possible factors that significantly differed between the remission and nonremission groups in the univariate analysis.

\section{Statistical Analysis}

Statistical analyses were performed using JMP Pro 13.2.1 (SAS Institute Inc., Cary, NC, USA). Categorical variables were presented as numbers and percentages and continuous variables as median and interquartile ranges. Data between the 2 groups were compared using the $\chi^{2}$ test for categorical variables and the MannWhitney $U$ test for numerical variables. Correlations between 2 continuous data were analyzed using the Spearman's correlation coefficient. The optimal cutoff values of continuous parameters for diagnosing nonremission after 8 weeks of treatment were determined via an ROC curve analysis. The independent risk factors for nonremission after 8 weeks of treatment were identified via a logistic regression analysis. A $p$ value of $<0.05$ was considered statistically significant.

\section{Results}

\section{Baseline Characteristics of the Patients}

Table 1 shows the baseline characteristics of the patients. The study included 29 (60.4\%) male patients. The median age of the patients was 36.5 years, and the median 
Table 1. Baseline characteristics of the patients

\begin{tabular}{ll}
\hline Variables & $\mathrm{UC}(n=48)$ \\
\hline Male sex, $n$ (\%) & $29(60.4)$ \\
Age, years & $36.5(22.5-43)$ \\
Disease duration, years & $0.5(0-3)$ \\
Disease extension, $n(\%)$ & \\
$\quad$ Proctitis & $0(0)$ \\
$\quad$ Left-sided colitis & $5(10.4)$ \\
$\quad$ Pan-colitis & $43(89.6)$ \\
Newly diagnosed or relapse, $n(\%)$ & \\
$\quad$ Newly diagnosed & $20(41.7)$ \\
$\quad$ Relapse & $28(58.3)$ \\
Mayo score & $8(7-9)$ \\
WBC count, $/ \mu \mathrm{L}$ & $8,350(6,250-10,075)$ \\
Hemoglobin level, $\mathrm{g} / \mathrm{dL}$ & $12.6(11.1-14.2)$ \\
Platelet count, $\times 10^{4} / \mu \mathrm{L}$ & $34.1(25.2-44.2)$ \\
Neutrophil count, $/ \mu \mathrm{L}$ & $5,467(3,716-8,403)$ \\
Lymphocyte count, $/ \mu \mathrm{L}$ & $6,270(988-5,735)$ \\
Albumin level, $\mathrm{g} / \mathrm{dL}$ & $3.7(3.2-3.9)$ \\
CRP level, mg/dL & $1.19(0.435-6.35)$ \\
NLR & $3.55(2.48-7.04)$ \\
PLR & $230.2(176.0-378.6)$ \\
\hline
\end{tabular}

Continuousvariableswere presentedas median and interquartile ranges. CRP, C-reactive protein; NLR, neutrophil-to-lymphocyte ratio; PLR, platelet-to-lymphocyte ratio.

disease duration was 0.5 years. Five (10.4\%) patients had left-sided colitis and 43 (89.6\%) had pan-colitis. In total, $20(41.7 \%)$ patients were newly diagnosed and 28 (58.3\%) had relapse. Twenty-four cases were treated with oral PSL, and 24 cases were treated with intravenous PSL. The median dose of PSL was $40 \mathrm{mg} /$ body (40-50 mg/body). The median Mayo score at baseline was 8 . The median NLR and PLR at baseline was 3.55 (2.48-7.04) and 230.2 (176.0-378.6), respectively.

\section{Differences in Baseline Parameters between the Remission and Nonremission Groups}

Of 48 patients, $28(58.3 \%)$ achieved remission after 8 weeks of treatment and 20 (41.7\%) did not. Table 2 depicts the baseline parameters between the remission and nonremission groups, as assessed via a univariate analysis. Sex, age, disease duration and extension, and treatment setting did not significantly differ between the 2 groups. The nonremission group had a significantly higher baseline Mayo score than the remission group $(p<$ $0.05)$. Among the baseline laboratory data, significant differences were found in terms of platelet and lymphocyte count, C-reactive protein (CRP) level, NLR, and PLR. The nonremission group had a higher platelet count $\left(\times 10^{4} /\right.$ $\mu \mathrm{L})$ than the remission group (39.7 [30.4-48.5] vs. 29.1 [24.3-38.6]; $p<0.05)$. The nonremission group had a lower lymphocyte count $(/ \mu \mathrm{L})$ than the remission group $(1,143[862-1,599]$ vs. $1,375[1,186-1,923] ; p<0.05)$. The nonremission group had a higher CRP level ( $\mathrm{mg} / \mathrm{dL})$ than the remission group $(4.45$ [1.48-9.13] vs. 0.80 [0.10$2.56] ; p<0.001)$. The NLR and PLR of the nonremission group were higher than those of the remission group (4.70 [3.04-11.3] vs. $3.10[1.36-16.42] ; p<0.05$, and 353.6 [220.3-499.8] vs. 207.2 [174.4-243.6]; $p<0.001$, respectively). Based on the ROC curve analysis, the optimal cutoff NLR and PLR for predicting nonremission was 3.6 and 262, respectively (Fig. 1).

\section{Independent Risk Factors for Predicting Nonremission} after 8 Weeks of Treatment

A multivariate analysis of pretreatment Mayo score, platelet and lymphocyte count, CRP level, NLR, and PLR, which are candidate predictors for nonremission after 8 weeks of treatment, was performed. The cutoff value of each parameter was determined via an ROC curve analysis. A Mayo score of $\geq 9$ (hazard ration [HR]: 14.83 , 95\% confidence interval [CI]: 1.88-117.2, $p=0.016)$, CRP level of $\geq 1.26 \mathrm{mg} / \mathrm{dL}$ (HR: 17.7, 95\% CI: 1.81-173.3, $p=$ 0.014), and PLR of $\geq 262$ (HR 23.1: 95\% CI: 1.29-413.7, $p$ $=0.033)$ were considered independent risk factors for nonremission after 8 weeks of treatment (Table 3).

\section{Discussion}

This study showed that both NLR and PLR were correlated with the therapeutic outcomes of systemic corticosteroid therapy for UC. Moreover, PLR was an independent predictor of nonremission after 8 weeks of treatment. To the best of our knowledge, this report first showed the efficacy of NLR and PLR as candidate biomarkers for predicting the therapeutic outcomes of systemic corticosteroid therapy in UC.

Systemic corticosteroid therapy is the standard induction-remission treatment for acute moderate or severe UC. However, about $45 \%$ of patients with UC treated with systemic corticosteroid are refractory to treatment [3]. Therefore, predicting the responsiveness to steroid therapy is important in preventing the continuation of ineffective treatment or in preparing for the second-line therapy or colectomy. Previous studies showed possible predictors of responsiveness to systemic corticosteroid. Baseline clinical factors, such as disease extent, stool frequency, temperature, heart rate, 
Table 2. Baseline parameters between the remission and nonremission groups

\begin{tabular}{lllc}
\hline & Remission group $(n=28)$ & Nonremission group $(n=20)$ & $p$ value \\
\hline Male sex, $n(\%)$ & $16(57.1)$ & $13(65.0)$ & 0.7656 \\
Age, years & $38(14-66)$ & $26.5(15-81)$ & 0.221 \\
Disease duration, years & $0.5(0-29)$ & $0.6(0-5)$ & 0.4090 \\
Left-sided colitis, $n(\%)$ & $1(3.6)$ & $4(20.0)$ & 0.1457 \\
Newly diagnosed, $n(\%)$ & $12(42.9)$ & $8(40.0)$ & 1.0000 \\
Mayo score & $7(6-8.75)$ & $9(8.25-10)$ & $<0.05^{*}$ \\
WBC count, $/ \mu \mathrm{L}$ & $1,900(5,400-9,925)$ & $9,000(7,300-11,375)$ & 0.2456 \\
Hemoglobin level, g/dL & $12.6(11.1-14)$ & $12.5(10.9-14.4)$ & 0.8836 \\
Platelet count, $\times 104 / \mu \mathrm{L}$ & $29.1(24.3-38.6)$ & $39.7(30.4-48.5)$ & $<0.05^{*}$ \\
Neutrophil count, $/ \mu \mathrm{L}$ & $5,116(9,285-9,016)$ & $7,474(5,842-3,800)$ & 0.1295 \\
Lymphocyte count, / $\mu \mathrm{L}$ & $7,375(6,186-4,923)$ & $7,143(862-1,599)$ & $<0.05^{*}$ \\
Albumin level, g/dL & $3.7(3.35-4.28)$ & $3.4(2.8-3.8)$ & 0.0957 \\
CRP level, $\mathrm{mg} / \mathrm{dL}$ & $0.80(0.103-2.56)$ & $4.45(1.48-9.13)$ & $<0.01^{*}$ \\
NLR & $3.10(2.26-4.33)$ & $4.70(3.04-11.3)$ & $<0.05^{*}$ \\
PLR & $207.2(174.4-243.6)$ & $353.6(220.3-499.8)$ & $<0.01^{*}$ \\
\hline
\end{tabular}

Continuous variables were presented as median and interquartile ranges. CRP, C-reactive protein; NLR, neutrophil-to-lymphocyte ratio; PLR, platelet-to-lymphocyte ratio. ${ }^{*} p<0.05$ indicates statistical significance.

Table 3. Independent risk factors for nonremission after 8 weeks of treatment based on a logistic regression analysis

\begin{tabular}{|c|c|c|c|}
\hline & Hazard ratio & $95 \% \mathrm{Cl}$ & $p$ value \\
\hline \multicolumn{4}{|c|}{ Mayo score } \\
\hline$<9$ & Ref & \multirow{2}{*}{$1.88-117.2$} & \multirow{2}{*}{$0.0106^{*}$} \\
\hline $9 \leq$ & 14.83 & & \\
\hline \multicolumn{4}{|c|}{ Platelet count } \\
\hline$<34.5$ & Ref & \multirow{2}{*}{$0.119-6.54$} & \multirow{2}{*}{0.9015} \\
\hline $34.5 \leq$ & 0.88 & & \\
\hline \multicolumn{4}{|c|}{ Lymphocyte count } \\
\hline$\geq 960$ & Ref & \multirow{2}{*}{$0.123-65.7$} & \multirow{2}{*}{0.5143} \\
\hline$<960$ & 2.84 & & \\
\hline \multicolumn{4}{|l|}{ CRP level } \\
\hline$<1.26$ & Ref & \multirow{2}{*}{$1.81-173.3$} & \multirow{2}{*}{$0.0135^{*}$} \\
\hline $1.26 \leq$ & 17.7 & & \\
\hline \multicolumn{4}{|l|}{ NLR } \\
\hline$<3.6$ & Ref & \multirow{2}{*}{$0.036-3.325$} & \multirow{2}{*}{0.3588} \\
\hline $3.6 \leq$ & 0.35 & & \\
\hline \multicolumn{4}{|l|}{ PLR } \\
\hline$<262$ & Ref & \multirow{2}{*}{$1.285-413.7$} & \multirow{2}{*}{$0.0332^{*}$} \\
\hline $262 \leq$ & 23.1 & & \\
\hline
\end{tabular}

95\% Cl, 95\% confidence interval; ref, reference; CRP, C-reactive protein; NLR, neutrophil-to-lymphocyte ratio; PLR, platelet-tolymphocyte ratio. ${ }^{*} p<0.05$ indicates statistical significance.

serum CRP and albumin levels, and radiologic assessment, were found to be the predictors of failed corticosteroid therapy in a previous systematic review [13]. According to the most recent guidelines for the man- agement of pediatric UC by the international Pediatric Inflammatory Bowel Disease Ahead Program (PIBDAhead), the clinical activity score on days 3 and 5 of hospital admission can predict the need for treatment escalation after intravenous corticosteroid therapy [14]. Moreover, the PIBD-Ahead guideline states that higher CRP levels on both days 3 and 5 of treatment can predict response to intravenous steroids [14]. The clinical activity and serum CRP levels are important factors affecting the outcomes of systemic corticosteroid therapy. The current study showed that a Mayo score of $\geq 9$ and a CRP level of $\geq 1.26 \mathrm{mg} / \mathrm{dL}$ were independent risk factors for nonremission after 8 weeks of treatment. These results are consistent with those of previous systematic review and the PIBD-Ahead guidelines.

We focused on the efficacy of NLR and PLR as candidate noninvasive predictors for the outcomes of systemic corticosteroid therapy in UC. NLR and PLR are simple biomarkers that can predict the disease activity of UC [49]. Recent studies have shown that NLR and/or PLR can be reliable markers for predicting the outcomes of tacrolimus and anti-TNF therapy in UC $[11,12]$. Therefore, whether NLR and PLR can be candidate markers for predicting response to systemic corticosteroids therapy in UC was evaluated. Results showed that the nonremission group had higher NLR and PLR than the remission group. Moreover, a PLR of $\geq 262$ at baseline was an independent risk factor for nonremission after 8 weeks of treatment. Hence, NLR and PLR can be noninvasive biomarkers for 
(A) Neutrophil-to-lymphocyte ratio (NLR)

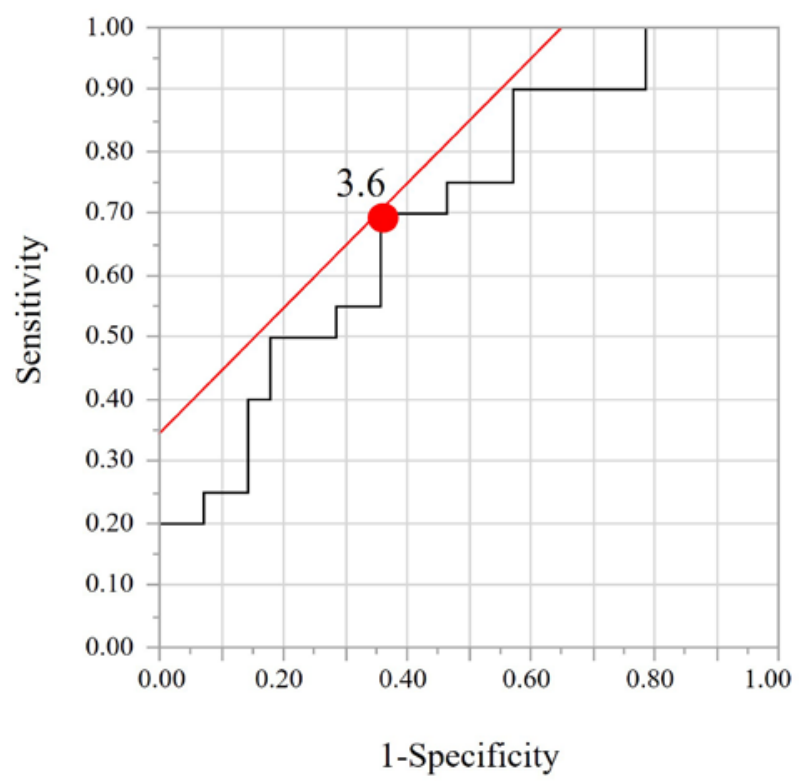

\section{(B) Platelet-to-lymphocyte ratio (PLR)}

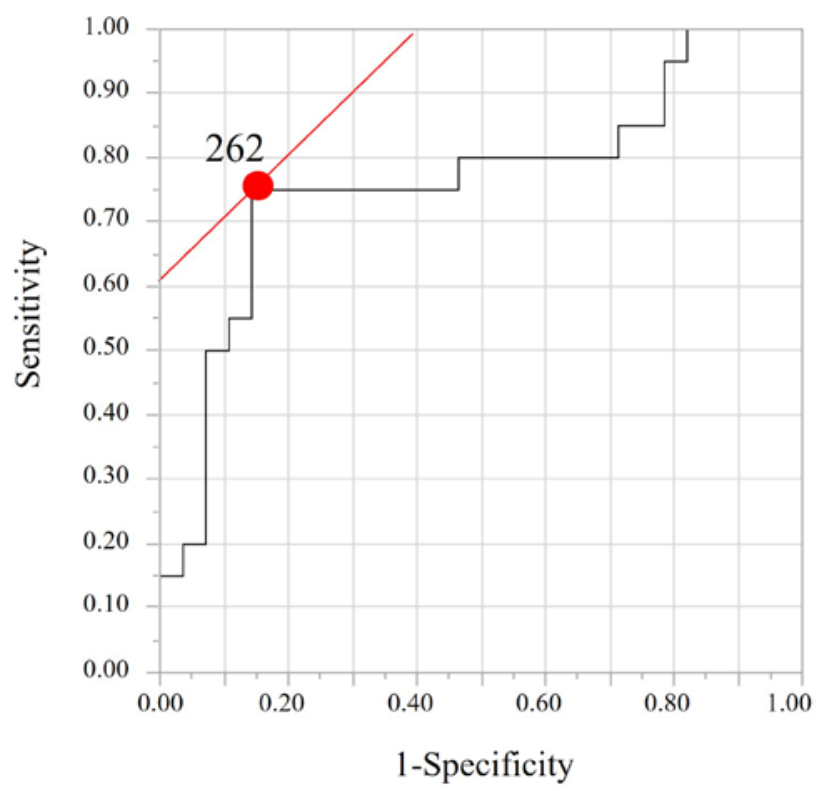

Fig. 1. ROC curve of NLR and PLR for predicting nonremission. A Based on the ROC curve analysis, the optimal cutoff NLR for predicting nonremission was 3.6 (sensitivity: 70.0\%, specificity: $64.3 \%$, and AUC: 0.702). B Based on the ROC curve analysis, the optimal cutoff PLR for predicting nonremission was 262 (sensitivity: $75.0 \%$, specificity: $85.7 \%$, and AUC: 0.764$)$. ROC, receiver operating characteristic; NLR, neutrophil-to-lymphocyte ratio; PLR, platelet-to-lymphocyte ratio; AUC, area under the curve. predicting the therapeutic outcomes of induction therapy with systemic corticosteroid in moderate or severe UC.

An important discussion point is the mechanism underlying the significant correlation between NLR and PLR as well as the therapeutic outcomes of systemic corticosteroid therapy in UC. Fundamentally, neutrophils induce systemic inflammation and play a significant role in the development of mucosal inflammatory conditions, such as cryptitis and crypt abscess formation, in UC. Moreover, a high platelet count and presence of activated platelets can exacerbate mucosal inflammation by increasing vascular permeability and by enhancing leukocyte migration ability in UC. In contrast, low lymphocyte counts are found in active UC, which may be associated with complex factors such as mucosal lymphocyte infiltration, apoptosis caused by autoimmune disorder, malnutrition, and leakage due to colonic bleeding. Thus, high neutrophil and platelet counts and a low lymphocyte count can reflect the immunological inflammatory intensity of UC. Therefore, NLR and PLR, which are obtained by dividing the neutrophil and platelet count by the lymphocyte count, are more sensitive in identifying the im- munological inflammatory intensity of UC than their respective values. Hence, NLR and PLR are excellent in predicting responsiveness to steroid treatment in UC.

Another discussion point is the difference between NLR, PLR, Mayo score, and CRP for predicting the corticosteroid therapeutic efficacy. Fundamentally, we consider NLR, PLR, Mayo score, and CRP to be markers for evaluating the disease activity of UC, each of which has an independent value. These markers can assess the disease intensity from different points of view. Mayo score consists of 4 subscores (stool frequency, rectal bleeding, findings on sigmoidoscopy, and physician's global assessment), which reflects mainly the clinical severity. CRP is an acute-phase protein that increases following interleukin- 6 secretion by macrophages and $\mathrm{T}$ cells, which reflects the intensity of systemic serological inflammation. NLR and PLR, which are obtained by dividing the neutrophil and platelet count by the lymphocyte count, can reflect the immunological inflammatory intensity of UC. Therefore, each marker has independent significance for assessing the disease activity and can predict the therapeutic outcome of corticosteroid therapy in UC from a 
different point of view. Thus, we argue that it is meaningful to predict treatment outcomes using NLR and PLR in addition to commonly used Mayo score and CRP. As for the difference between NLR and PLR, it has not been clarified even in terms of its ability to assess disease activity in UC. The previous study has reported that both NLR and PLR can predict mucosal healing in patients with UC treated with anti-TNF antibodies [12]. Of the NLR and PLR, only PLR was identified as significant for predicting therapeutic outcomes of corticosteroid therapy in the multivariate analysis in our study. In the present study, the case number is too small to analyze the difference between NLR and PLR. Further investigation is needed to clarify this issue.

The current study had several limitations. That is, it was retrospective in nature, and only a single-center cohort was included. Moreover, endoscopic remissions were not evaluated in this study. Endoscopic activities were evaluated before the systemic corticosteroid therapy in all cases enrolled in this study. However, only a few cases were evaluated endoscopically after 8 weeks of the treatment. Ideally, a prospective study should be conducted to investigate the correlation between pretreatment NLR and PLR and endoscopic improvement after systemic corticosteroid therapy. The most important limitation is the inclusion of a small number of patients. Thus, further studies with a large cohort should be performed to validate the predictive value of NLR and PLR for the efficacy of systemic corticosteroid in UC.

In conclusion, pretreatment NLR and PLR were significantly associated with clinical remission after 8 weeks of induction therapy with systemic corticosteroid in patients with UC. Besides a high Mayo score and high CRP level, high PLR was identified as an independent risk factor for nonremission after 8 weeks of systemic corticosteroid therapy. These results indicate that NLR and PLR can be noninvasive biomarkers for predicting the therapeutic outcomes of induction therapy with systemic corticosteroid in UC.

\section{Statement of Ethics}

All procedures followed have been performed in accordance with the ethical standards laid down in the 1964 Declaration of Helsinki and its later amendments. The study design was observational. The study was approved by the ethics committee of our institution (Institutional Review Board of the Tohoku Medical and Pharmaceutical University School of Medicine). Approved IRB number is 2021-2-036. The study was performed in accordance with the ethics guidelines in Japan and was allowed implementation without obtaining individual written informed consent. This study was announced by posters in the outpatient ward or on the homepage of the participating institutions. Patients who expressed unwillingness to participate in this study were excluded.

\section{Conflict of Interest Statement}

The authors declare no conflicts of interest.

\section{Funding Sources}

The authors received no financial support for the research.

\section{Author Contributions}

Katsuya Endo established the study design, and he was involved in designing the original study protocol, collecting data, and writing the manuscript. Tomonori Satoh, Yuki Yoshino, Shiho Kondo, Yoko Kawakami, and Tomofumi Katayama participated in data collection. Yoshiteru Sasaki, Atsuko Takasu, Takayuki Kogure, Morihisa Hirota, Takayoshi Meguro, and Kennichi Satoh contributed in discussing and critically reviewing the manuscript.

\section{Data Availability Statement}

The data that support the findings of this study are available from the corresponding author upon reasonable request.

\section{References}

1 Matsuoka K, Kobayashi T, Ueno F, Matsui T, Hirai F, Inoue N, et al. Evidence-based clinical practice guidelines for inflammatory bowel disease. J Gastroenterol. 2018;53(3):305-53.

2 Harbord M, Eliakim R, Bettenworth D, Karmiris K, Katsanos K, Kopylov U, et al. Third European evidence-based consensus on diagnosis and management of ulcerative colitis. Part 2: current management. J Crohns Colitis. 2017;11(7):769-84.
3 Faubion WA Jr, Loftus EV Jr, Harmsen WS, Zinsmeister AR, Sandborn WJ. The natural history of corticosteroid therapy for inflammatory bowel disease: a population-based study. Gastroenterology. 2001;121(2):255-60.

4 Torun S, Tunc BD, Suvak B, Yildiz H, Tas A, Sayilir A, et al. Assessment of neutrophil-lymphocyte ratio in ulcerative colitis: a promising marker in predicting disease severity. Clin Res Hepatol Gastroenterol. 2012;36(5):491-7.
5 Celikbilek M, Dogan S, Ozbakır O, Zararsız G, Kücük H, Gürsoy S, et al. Neutrophil-lymphocyte ratio as a predictor of disease severity in ulcerative colitis. J Clin Lab Anal. 2013; 27(1):72-6.

6 Demir AK, Demirtas A, Kaya SU, Tastan I, Butun I, Sagcan M, et al. The relationship between the neutrophil-lymphocyte ratio and disease activity in patients with ulcerative colitis. Kaohsiung J Med Sci. 2015;31(11):585-90.
Neutrophil-to-Lymphocyte and Plateletto-Lymphocyte Ratios in UC
Inflamm Intest Dis 2021;6:218-224 DOI: $10.1159 / 000520523$ 
7 Akpinar MY, Ozin YO, Kaplan M, Ates I, Kalkan IH, Kilic ZMY, et al. Platelet-to-lymphocyte ratio and neutrophil-to-lymphocyte ratio predict mucosal disease severity in ulcerative colitis. J Med Biochem. 2018;37(2): $155-62$.

8 Okba AM, Amin MM, Abdelmoaty AS, Ebada HE, Kamel AH, Allam AS, et al. Neutrophil/lymphocyte ratio and lymphocyte/ monocyte ratio in ulcerative colitis as noninvasive biomarkers of disease activity and severity. Auto Immun Highlights. 2019; 10(1):4.

9 Jeong Y, Jeon SR, Kim HG, Moon JR, Lee TH, Jang JY, et al. The role of platelet to lymphocyte ratio and neutrophil to lymphocyte ratio in ulcerative colitis. Intest Res. 2021;19(1): 62-70.

10 Nishida Y, Hosomi S, Yamagami H, Yukawa T, Otani K, Nagami Y, et al. Neutrophil-tolymphocyte ratio for predicting loss of response to infliximab in ulcerative colitis. PLoS One. 2017;12(1):e0169845.

11 Nishida Y, Hosomi S, Yamagami H, Sugita N, Itani S, Yukawa T, et al. Pretreatment neutrophil-to-lymphocyte ratio predicts clinical relapse of ulcerative colitis after tacrolimus induction. PLoS One. 2019;14(3):e0213505.

12 Bertani L, Rossari F, Barberio B, Demarzo MG, Tapete G, Albano E, et al. Novel prognostic biomarkers of mucosal healing in ulcerative colitis patients treated with anti-
TNF: neutrophil-to-lymphocyte ratio and platelet-to-lymphocyte ratio. Inflamm Bowel Dis. 2020;26(10):1579-87.

13 Turner D, Walsh CM, Steinhart AH, Griffiths AM. Response to corticosteroids in severe ulcerative colitis: a systematic review of the literature and a meta-regression. Clin Gastroenterol Hepatol. 2007;5(1):103-10.

14 Orlanski-Meyer E, Aardoom M, Ricciuto A, Navon D, Carman N, Aloi M, et al. Predicting outcomes in pediatric ulcerative colitis for management optimization: systematic review and consensus statements from the pediatric inflammatory bowel disease-ahead program. Gastroenterology. 2021;160(1): 378-402.e22. 